\title{
The Effect of Tamoxifen Treatment During 24 Months On Alkaline Phosphatase Concentration In Breast Cancer Patients
}

\author{
Betul Akcesme \\ International University of Sarajevo, Faculty of Engineering and Natural Sciences \\ Hrasnicka Cesta 15, 71000 Sarajevo, Bosnia and Herzegovina \\ bcicek@ius.edu.ba
}

\begin{abstract}
Determination of alkaline phosphatase (ALP) isoenzyme activity is useful for the diagnosis and clinical evaluation of cancer patients, including breast cancer. 21 women were randomized to receive tamoxifen, probably the most widely used endocrine treatment for breast cancer worldwide, for 2 years, and 21 women constituted the control group. Control group were treated without Tamoxifen considering placebo effect. Alkaline phosphatase level was significantly lower in tamoxifen treated female breast cancer patients when compared with those of the treated patients with placebo. Results indicates that the tamoxifen decrease the level of ALP in first three months and then stabilize this level in the following months.
\end{abstract}

Key words: Breast Cancer, Tamoxifen, Alkaline Phosphatase

\section{INTRODUCTION}

Breast cancer is the most common cancer in the female population which originating from breast tissue, most commonly from the inner lining of milk ducts or the lobules that supply the ducts with milk. [1]. Even though great progress in the treatment of breast cancer has been achieved during past decades, it is still the principal cause of cancer death among women worldwide [2,3].

Alkaline phosphatase (ALP) comprises a group of isoenzymes. These enzymes have some important function like hydrolyzing of phosphate esters in an alkaline environment. They generate an inorganic phosphate and organic radicals [4].The total serum alkaline phosphatase activity represents several isoenzymes in different organs. ALP is a sensitive indicator of mild biliary obstruction, thus being a very sensitive indicator of liver progression in cancer patients. [5] Manys studies reveal that the determination of alkaline phosphatase (ALP) isoenzyme activity is useful for the diagnosis and clinical evaluation of cancer patients. Some other studies have shown increased activities of alkaline phosphatase (ALP) in patients with malignancy including breast cancer. [6,7].

There are different breast cancer types which require specific treatments. One of the possible treatments for breast cancer is hormone therapy. Hormone-sensitive breast cancer cells contain proteins known as hormone receptors that become activated when hormones bind to them. The activated receptors cause changes in the expression of specific genes, which can lead to the stimulation of cell growth. If the tumor cells contain estrogen receptors, the cancer is called estrogen receptorpositive (ER-positive), estrogen-sensitive, or estrogen-responsive. Hormone therapy (also called hormonal therapy, hormone treatment, or endocrine therapy) slows or stops the growth of hormonesensitive tumors by blocking the body's ability to produce hormones or by interfering with hormone 
action. One of the hormone therapies is to block estrogen's effects: Several types of drugs interfere with estrogen's ability to stimulate the growth of breast cancer cells: Selective estrogen receptor modulators(SERMs) bind o estrogen receptors, preventing estrogen from binding. Examples of SERMs approved by the Food and drug administration (FDA) are tamoxifen (Nolvadex ${ }^{\circledR}$ ), raloxifene $\left(\right.$ Evista $^{\circledR}$ ), and toremifene (Fareston ${ }^{\circledR}$ ).

[8] Tamoxifen, probably the most widely used endocrine treatment for breast cancer worldwide, has been used for more than 30 years. Tamoxifen has been approved by the FDA for adjuvant hormone treatment of premenopausal and postmenopausal women (and men) with ERpositive early-stage breast cancer. [9]

Since the ALP level in breast cancer patients is proper marker, this study aims to analyze the change of ALP level with the tamoxifen treatment in order to see tamoxifen effect on ALP level.

\section{MATERIALS AND METHODS}

21 women were randomized to receive tamoxifen $30 \mathrm{mg} / \mathrm{d}$ for 2 years, and 21 women constituted the control group. Control group were treated without Tamoxifen considering placebo effect. Inclusion criteria were operation for low-risk breast cancer and cessation of menstruations for more than 1 year. Exclusion criteria were presence of metastases, disorders of bone metabolism contraindications against tamoaxifen, use of drugs with influence on bone metabolism, ailments that made bone mineral measurements impossible, and age greater than 65 years. [6]

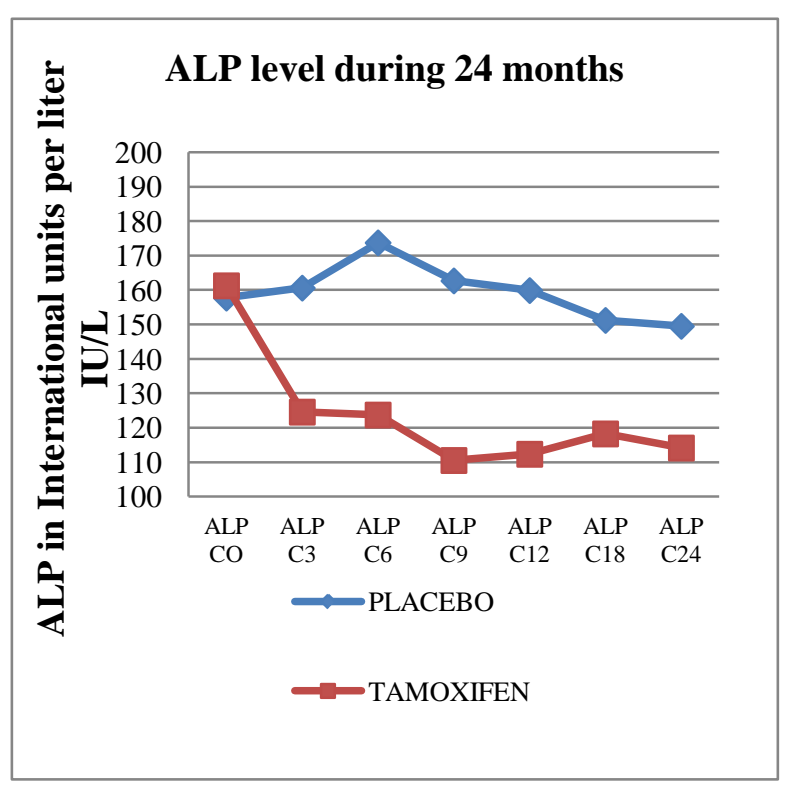

Figure 1. The level of ALP during 24 months with and without tamoxifen treatment
Repeated measurements of serum alkaline phosphatase (ALP) were performed in all patients. (ALP in International units per liter IU/L).

\subsection{Statistical Analyzes}

First, the treated patients with placebo and tamoxifen considered as two separated groups and their alkaline phosphatase level were compared and the two treatment effects were analyzed in 24 months period. Since the patient's alkaline phosphatase levels are measured repeatedly, the tamoxifen effects on alkaline phosphate level were analyzed both between factors; tamoxifen and placebo and within factor; 7 times (each 3 months in 2 years) with Two-way Repeated Measures Analysis of Variance (ANOVA)using Office 2007Excel. Furthermore, each time periods were subjected to $\mathrm{t}$ test: two sample assuming equal variance in order to see the changes in the level of ALP during the time in given interval, also with using Office 2007-Excel.

\section{RESULTS AND DISCUSSION}

Two-way Repeated Measures Analysis of Variance showed that alkaline phosphatase level were significantly lower in tamoxifen treated female breast cancer patients when compared with those of the treated patients with placebo (at $\mathrm{p}<0.05$, $\mathrm{p}=1,71 \mathrm{E}-10)$. When it is considered that all patients treated (with and without tamoxifen) alkaline phosphatase levels do not change with time $(p<0.05, \mathrm{p}=0.43)$. Although there is significant difference between treated and placebo group analyzes revealed that there is no significant changes during the indicated periods. The subjected data requires one more analysis to find the reason of these significant differences between alkaline phosphatase in two groups.

Each group ( tamoxifen treated and control) alkaline phosphatase levels in each time period subjected to T-test two sample assuming equal variances $(p>0.05)$ the result reveal that the tamoxifen treated patients shows significant difference in the level of ALP between baseline and at 3 months ( $p>0.05, p=0.001)$ and there is no significant differences between other periods. Results indicates that the tamoxifen decrease the level of ALP in three months and then stabilize this level in the following months as shown in figure 1.

\section{CONCLUSION}

Developments in breast cancer treatment are very essential, since it is one of the most common cancers in the female population. As statistical tests reveal that tamoxifen treatment has big effect on decreasing the level of alkaline phosphatase which is good indicator of breast cancer. It is proven that 
first 3 months of treatment is very effective to decrease ALP level with tamoxifen and after 3 months it is stabilized. There is need for further researches to determine the most effective treatment schedule with tamoxifen.

\section{REFERENCES}

1. Sariego J. Breast cancer in the young patient. Am Surg. 2010 Dec;76(12):1397400.

2. Jemal A, Siegel R, Ward E, Hao Y, Xu J, Thun MJ. Cancer statistics.CA Cancer J Clin. 2009; 59:225-249.

3. Goldhirsch A, Ingle JN, Gelber RD, Coates AS, Thurlimann B, Senn HJ.Thresholds for therapies: highlights of the St Gallen

4. International Expert Consensus on the primary therapy of early breast cancer. Ann Oncol. 2009; 20:1319-1329. Woodhouse A.C, Chuaqui R.F, Liotta L.A. Cancer (80) 1997; 1529-1537.

5. Crivellari D, Price KN, Hagen $M$ et al. Routine tests during follow-up of patients after primary treatment for operable breast cancer. International (Ludwig) Breast

6. Kristensen B, Ejlertsen B, Dalgaard P, Larsen L, Holmegaard SN, Transbøl I, Mouridsen HT Department of Clinical Physiology, Rigshospitalet, Copenhagen, Denmark. Tamoxifen and bone metabolism in postmenopausal low-risk breast cancer patients: a randomized study. [1994, 12(5):992-997]

7. B Prabasheela, S Baskaran, R Arivazhaga, Evaluation of alkaline phosphatase in pre and post operative breast cancer patients, Int J Biol Med Res. 2012; 3(2): 1536-1537

8. Wiwanitkit V . High Serum alkaline phosphatase level, a study in 181 Thai adult hospitalized patients. BMC Fam Pract. 2001; 2: 2.

9. Guidance for the management of breast cancer treatment-induced bone loss: a consensus position statement from a UK Expert Group. Cancer Treat Rev 2008;34:S1-S18. 